\title{
Electroweak non-resonant corrections to top pair production close to threshold
}

\author{
M. Beneke ${ }^{a}$, B. Jantzen ${ }^{a}$ and P. Ruiz-Femenía* $a b \dagger$ \\ a Institut für Theoretische Teilchenphysik und Kosmologie, RWTH Aachen University \\ D-52056 Aachen, Germany \\ ${ }^{b}$ Fakultät für Physik, Universität Wien, 1090 Wien, Austria \\ E-mail: ruiz@physik.rwth-aachen.de
}

\begin{abstract}
The production of $W^{+} W^{-} b \bar{b}$ from $e^{+} e^{-}$collisions at energies close to the $t \bar{t}$ threshold is dominated by the resonant process with a nearly on-shell $t \bar{t}$ intermediate state. The $W b$ pairs in the final state can also be reached through the decay of off-shell tops or through background processes containing no or only single top quarks. This non-resonant production starts to contribute at NLO to the $W^{+} W^{-} b \bar{b}$ total cross section in the non-relativistic power-counting $v \sim \alpha_{s} \sim \sqrt{\alpha_{E W}}$. The NLO non-resonant corrections presented in this talk represent the non-trivial NLO electroweak corrections to the $e^{+} e^{-} \rightarrow W^{+} W^{-} b \bar{b}$ cross section in the top anti-top resonance region. In contrast to the QCD corrections which have been calculated (almost) up to NNNLO, the parametrically larger NLO electroweak contributions have not been completely known so far, but are mandatory for the required accuracy at a future linear collider. We consider the total cross section of the $e^{+} e^{-} \rightarrow W^{+} W^{-} b \bar{b}$ process and additionally implement cuts on the invariant masses of the $W^{+} b$ and $W^{-} \bar{b}$ pairs.
\end{abstract}

35th International Conference of High Energy Physics - ICHEP2010,

July 22-28, 2010

Paris France

\footnotetext{
* Speaker.

†Preprint numbers: TTK-10-53, SFB/CPP-10-124. This work is supported by the DFG Sonderforschungsbereich/Transregio 9 "Computergestützte Theoretische Teilchenphysik".
} 


\section{Introduction}

The top-quark mass is currently known from direct production at the Fermilab Tevatron (and soon at the Large Hadron Collider) with a precision $\gtrsim 1 \mathrm{GeV}$. From a threshold scan of the $e^{+} e^{-} \rightarrow$ $t \bar{t}$ cross section at the planned International Linear Collider (ILC), however, an order of magnitude improvement in the precision can be achieved experimentally [1]. Aside from determining a fundamental parameter of the Standard Model, accurate top-mass measurements constrain the quantum fluctuations from non-standard interactions in electroweak precision measurements. Other characteristics of the top quark such as its width and Yukawa coupling provide information about its coupling to other particles and the mechanism of electroweak symmetry breaking. For these reasons top-quark pair production near threshold in $e^{+} e^{-}$annihilation has been thoroughly investigated following the non-relativistic QCD (NRQCD) approach, which treats the leading colour-Coulomb force exactly to all orders in perturbation theory. In this framework, where the strong coupling $\alpha_{s}$ is of the same order as $v$, the small relative velocity of the top and anti-top, most QCD corrections to the total cross section have been calculated up to NNNLO (see the summary [2]), and next-to-next-to-leading logarithms of $v$ have been partially summed [3].

Here we focus on the subleading electroweak corrections, which have received much less attention. The top quark is unstable with a significant width $\Gamma_{t}$ of about $1.5 \mathrm{GeV}$ due to the electroweak interaction. The width is essential in threshold production, since it prevents the top and anti-top from forming a bound state and causes a broad resonance structure in the energy dependence of the cross section on top of the increase due to the opening-up of the two-particle phase space. Once the top width is included, due to top decay, the physical final state is $W^{+} W^{-} b \bar{b}-$ at least if we neglect the decay of top into strange and down quarks, as justified by $V_{t b} \approx 1$, and consider $W$ bosons as stable. The $W^{+} W^{-} b \bar{b}$ final state can also be produced non-resonantly, i.e. through processes which do not involve a nearly on-shell $t \bar{t}$ pair. The latter effects are not included in the standard non-relativistic treatment. Adopting a counting scheme where $\alpha_{\mathrm{EW}} \sim \alpha_{s}^{2}$, we find that the leading non-resonant and off-shell effects are NLO for the total cross section, since there is an additional power of $\alpha_{\mathrm{EW}}$ but no phase-space suppression, hence the relative correction is $\alpha_{\mathrm{EW}} / v \sim \alpha_{s}$. Purely resonant electroweak effects, on the other hand, yield NNLO corrections [4].

In this talk we present the calculation of the non-resonant NLO electroweak contributions to the $e^{+} e^{-} \rightarrow W^{+} W^{-} b \bar{b}$ process in the $t \bar{t}$ resonance region, for the total cross section as well as including invariant-mass cuts on the $W b$ pairs. The calculation is performed with unstable-particle effective field theory [5], which provides the framework for consistently including resonant and non-resonant effects while maintaining an expansion in the small parameters of the problem.

\section{Unstable-particle effective theory for pair production near threshold}

The cross section for the $e^{+} e^{-} \rightarrow W^{+} W^{-} b \bar{b}$ process is obtained from the $W^{+} b W^{-} \bar{b}$ cuts of the $e^{+} e^{-}$forward-scattering amplitude. In the energy region $\sqrt{s} \approx 2 m_{t}$ the amplitude is dominated by the production of resonant top quarks with small virtuality. This allows us to integrate out hard modes $\left(\sim m_{t}\right)$ and represent the forward-scattering amplitude as the sum of two terms [5],

$$
i \mathscr{A}=\sum_{k, l} C_{p}^{(k)} C_{p}^{(l)} \int d^{4} x\left\langle e^{-} e^{+}\left|\mathrm{T}\left[i \mathscr{O}_{p}^{(k) \dagger}(0) i \mathscr{O}_{p}^{(l)}(x)\right]\right| e^{-} e^{+}\right\rangle+\sum_{k} C_{4 e}^{(k)}\left\langle e^{-} e^{+}\left|i \mathscr{O}_{4 e}^{(k)}(0)\right| e^{-} e^{+}\right\rangle .(2
$$



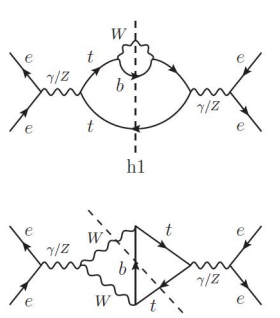

h3

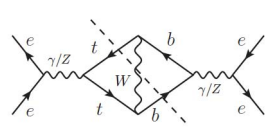

h2

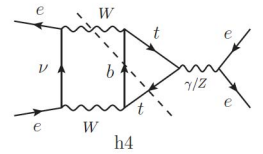

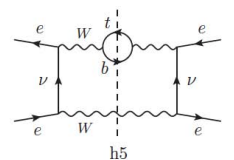
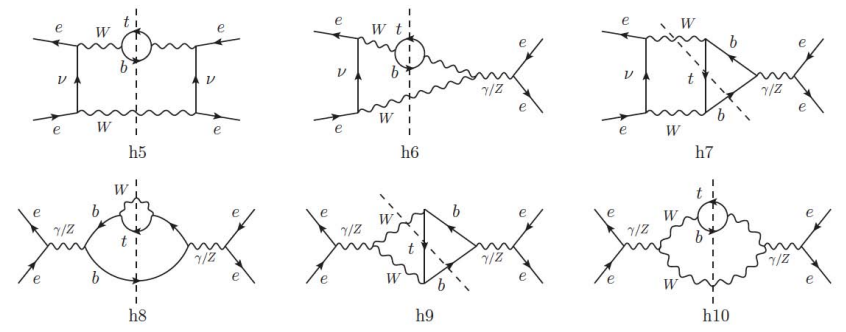

Figure 1: Two-loop forward-scattering amplitude diagrams with $\bar{t} b W^{+}$cuts. $t \bar{b} W^{-}$cuts and symmetric diagrams are not shown. The contribution to the $W^{+} W^{-} b \bar{b}$ cross section from diagrams $h_{1}-h_{10}$ can be interpreted as the $\bar{b} W^{-}$pair originating from a nearly on-shell anti-top decay, while the $b W^{+}$pair is produced non-resonantly, either from a highly virtual top (diagrams $\left.h_{1}-h_{4}\right)$, or without an intermediate top $\left(h_{5}-h_{10}\right)$.

The matrix elements in (2.1) are evaluated in the "low-energy" effective theory, which includes elements of soft-collinear and non-relativistic effective theory. The first term on the right-hand side of (2.1) describes the production of a resonant $t \bar{t}$ pair in terms of production (decay) operators $\mathscr{O}_{p}^{(l)}(x)\left(\mathscr{O}_{p}^{(k) \dagger}(x)\right)$ with short-distance coefficients $C_{p}^{(k, l)}$. The second term accounts for the remaining non-resonant contributions, which in the effective theory are described by four-electron production-decay operators $\mathscr{O}_{4 e}^{(k)}$. The calculation of the short-distance coefficients $C_{4 e}^{(k)}$ is performed in standard fixed-order perturbation theory in the full electroweak theory. In particular, the top propagator is the free one not including the top width, which ensures that the amplitude depends only on the short-distance scales. The leading imaginary parts of $C_{4 e}^{(k)}$ arise from the cut two-loop diagrams of order $\alpha_{\mathrm{EW}}^{3}$ shown in Fig. 1. The corresponding contribution to the cross section is

$$
\sigma_{\text {non-res }}=\frac{1}{s} \sum_{k} \operatorname{Im}\left[C_{4 e}^{(k)}\right]\left\langle e^{-} e^{+}\left|i \mathscr{O}_{4 e}^{(k)}(0)\right| e^{-} e^{+}\right\rangle .
$$

Technically, this simply amounts to the calculation of the spin-averaged tree-level processes $e^{+} e^{-} \rightarrow$ $t W^{-} \bar{b}$ and $e^{+} e^{-} \rightarrow \bar{t} W^{+} b$ with no width supplied to the intermediate top-quark propagators. Instead, the divergence from the top-quark propagators going on-shell is regularized dimensionally. Details on the computation and integral representations of the result for (2.2) can be found in [6].

Through the computation of the four-electron matching coefficients loose cuts $\left(\sim m_{t}\right)$ on the $b W^{+}$and $\bar{b} W^{-}$invariant masses can be incorporated easily, as it has been discussed in the context of $W$-pair production near threshold [7]. The result obtained in [6] covers the case of symmetric cuts on the invariant mass of the $b W$ subsystems $\left(p_{b W}^{2}\right)$ of the form $m_{t}-\Delta M_{t} \leq \sqrt{p_{b W}^{2}} \leq m_{t}+\Delta M_{t}$, for $\Delta M_{t} \gg \Gamma_{t}$, up to the total cross section $\left(\Delta M_{t, \max }=m_{t}-M_{W}\right)$. An alternative approach has been developed in parallel $[8,9]$ that includes the effects of invariant-mass cuts on the $W b$ pairs entirely through calculations in NRQCD. This works if the invariant-mass cuts around $m_{t}$ are neither very loose nor very tight, and provided that the non-resonant background processes are small (which at NLO was checked [9] by computing the full $e^{+} e^{-} \rightarrow W^{+} W^{-} b \bar{b}$ cross section at tree-level with MadGraph). Under these assumptions, part of the $\alpha_{s}$-corrections to the non-resonant contributions has already been analyzed in [9], which in our approach correspond to NNLO contributions.

\section{Results}

The left plot in Fig. 2 displays the relative sizes of the NLO electroweak corrections with respect to the $\mathrm{LO}$ result for the $e^{+} e^{-} \rightarrow W^{+} W^{-} b \bar{b}$ cross section, which includes the summation of Coulomb corrections. The QED contribution represents a correction of about $2 \%$ above threshold 

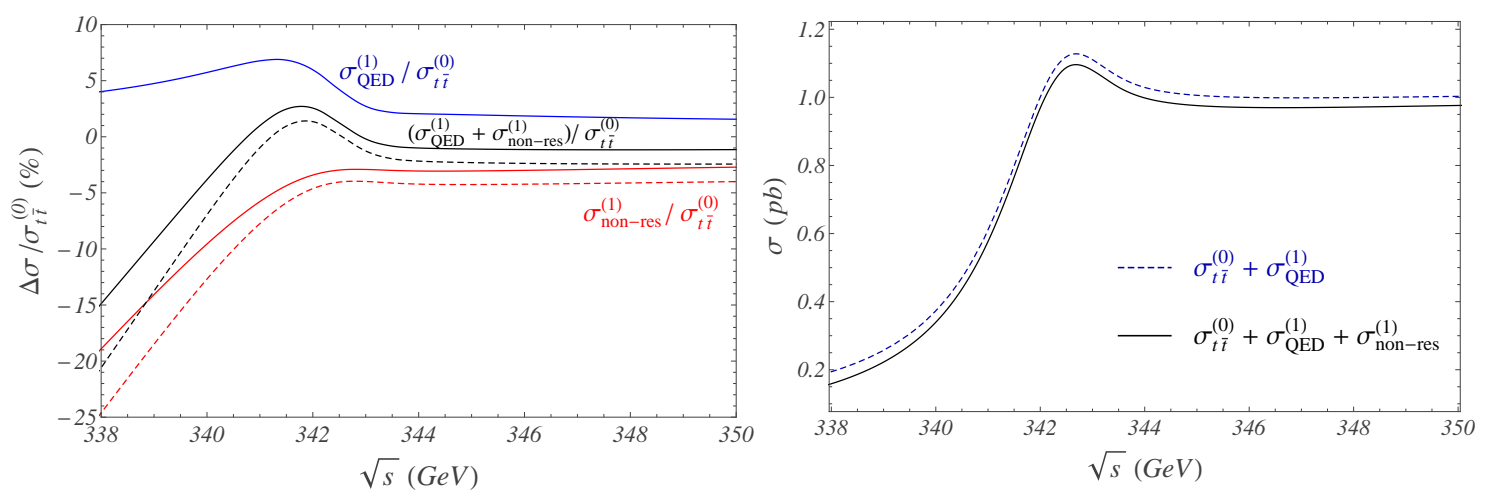

Figure 2: Left: Relative sizes of the QED, $\sigma_{\mathrm{QED}}^{(1)}$, and non-resonant, $\sigma_{\text {non-res }}^{(1)}$, corrections with respect to the LO cross section, $\sigma_{t \bar{t}}^{(0)}$, in percent, for $\Delta M_{t \text {, max }}$ (solid) and $\Delta M_{t}=15 \mathrm{GeV}$ (dashed). Right: Total cross section with LO QCD effects (dashed) and including NLO electroweak corrections (solid) at energies close to threshold. Input parameters: $m_{t}=172 \mathrm{GeV}, \Gamma_{t}=1.47 \mathrm{GeV}$ and $\alpha_{s}(30 \mathrm{GeV})=0.142$.

and rises to a maximum of $7 \%$ just below the peak, while the non-resonant contributions give a constant negative shift of about 3\% above threshold. Below threshold the relative size of the nonresonant corrections is very large, since the LO result rapidly vanishes, reaching up to $19 \%$. Hence below threshold they represent the leading electroweak correction to the total $t \bar{t}$ cross section. We observe a partial cancellation of the QED and non-resonant corrections in the peak region and at energies above. A sensitivity to the invariant-mass cut $\Delta M_{t}$ in the $b W^{+}$and $\bar{b} W^{-}$subsystem enters first at NLO through the non-resonant contributions. Restricting the available phase-space for the final-state particles by tightening the invariant-mass cuts $\Delta M_{t}$ makes the non-resonant contributions even more important. This is shown by the dashed lines in Fig. 2, corresponding to $\Delta M_{t}=15 \mathrm{GeV}$.

Aside from the pure QCD corrections, the NLO prediction for the $e^{+} e^{-} \rightarrow W^{+} W^{-} b \bar{b}$ total cross section is displayed by the solid line in the right plot of Fig. 2. The absolute size of the non-resonant correction is given by the difference between the dashed line, which only includes the QED NLO correction, and the solid one. This negative shift amounts to 27-35 fb for $\sqrt{s}$ in the interval $(338,350) \mathrm{GeV}$.

\section{References}

[1] M. Martinez and R. Miquel, Eur. Phys. J. C27, 49 (2003).

[2] M. Beneke, Y. Kiyo and K. Schuller, PoS RADCOR2007, 051 (2007).

[3] A. H. Hoang, A. V. Manohar, I. W. Stewart and T. Teubner, Phys. Rev. D65, 014014 (2002); A. Pineda and A. Signer, Nucl. Phys. B762, 67 (2007).

[4] A. H. Hoang and C. J. Reisser, Phys. Rev. D 71, 074022 (2005).

[5] M. Beneke, A. P. Chapovsky, A. Signer and G. Zanderighi, Phys. Rev. Lett. 93, 011602 (2004); M. Beneke, A. P. Chapovsky, A. Signer and G. Zanderighi, Nucl. Phys. B686, 205 (2004); M. Beneke, P. Falgari, C. Schwinn, A. Signer and G. Zanderighi, Nucl. Phys. B 792, 89 (2008).

[6] M. Beneke, B. Jantzen and P. Ruiz-Femenia, Nucl. Phys. B 840, 186 (2010).

[7] S. Actis, M. Beneke, P. Falgari and C. Schwinn, Nucl. Phys. B 807, 1 (2009).

[8] A. H. Hoang, C. J. Reisser and P. Ruiz-Femenia, Nucl. Phys. Proc. Suppl. 186, 403 (2009).

[9] A. H. Hoang, C. J. Reisser and P. Ruiz-Femenia, Phys. Rev. D 82, 014005 (2010). 